\title{
Laryngeal framework surgery
}

\begin{abstract}
Laryngeal framework surgery is defined as surgical procedures performed on the laryngeal skeleton and the insertion of muscles to correct vocal fold positioning and tension. Its main objective is to improve the voice without directly intervening in the vocal folds. The European Society of Laryngology proposed in 2000 a classification and nomenclature of these surgeries according to their purpose. Such standardization has divided these procedures into four groups: Approximation laryngoplasty, Expansion laryngoplasty, Relaxation laryngoplasty, and Tensioning laryngoplasty. Indications, techniques, and complications of each procedure will be described in this review.
\end{abstract}

Keywords: Thyroplasty, presbyphonia, dysphonia
Volume 12 Issue 5 - 2020

\author{
Guilherme Simas do Amaral Catani,' Maria \\ Eduarda Carvalho Catani, ${ }^{2}$ Letícia Raysa \\ Schiavon Kinasz,' Gabriela Alves Marroni,' \\ Marcelly Botelho Soares,' Patrícia Cristina \\ Scarabotto,' Rogério Hamerschmidt ${ }^{\prime}$ \\ 'Federal University of Paraná, Brazil \\ ${ }^{2}$ UNIFEBE Medical School, Brusque, Brazil \\ ${ }^{3}$ UNICAMP, Campinas, Brazil
}

Correspondence: Guilherme Simas do Amaral Catani, Department of Otorhinolaryngology, Rua Conselheiro Araújo 90 cj 7I, Curitiba Paraná, Brasil 80060-230,

Tel \&fax+55413262671।, Email gscani@gmail.com

\section{Introduction}

The first description of surgery involving the laryngeal framework dates from 1915 by Payr, but this type of procedure became popular through the Isshiki techniques from $1970{ }^{1,2}$ This is one of the most dynamic areas of phonosurgery and its main objective is to improve the voice without directly intervening in the vocal folds. ${ }^{1-3}$ The European Society of Laryngology proposed in 2000 a classification and nomenclature of these surgeries according to their purpose. ${ }^{1,4}$ Such standardization has divided these procedures into four groups, which will be detailed in this review.Laryngeal framework surgery is defined as surgical procedures performed on the laryngeal skeleton and/or the insertion of muscles to correct vocal fold positioning and/ or tension. ${ }^{1}$

They are performed under local anesthesia and light sedation to allow monitoring of the voice. ${ }^{2}$ Indirect laryngoscopy through flexible fiber is recommended during the procedure to visualize the intralaryngeal aspect. ${ }^{4}$ An extremely important factor for the performance of this surgery is the knowledge and the marking of the reference points from the skin incision to the realization of the window in the thyroid cartilage. The first step is to place a cushion under the shoulders to allow the neck to be hyperextended and to orientate the patient about this type of position, as it can be uncomfortable.

\section{Approximation laryngoplasty}

Indicated for cases of insufficient glottic closure such as vocal fold paralysis, presbyphonia, vocal fold atrophy, glottictumor resection, spasmodic abduction dysphonia, among others. ${ }^{1,2,5-7}$ Of the laryngeal framework surgeries, this one group is by far the most accomplished. ${ }^{2,8}$

\section{Medializationthyroplasty (type I)}

After positioning the patient, the thyroid cartilage is palpated so that the incision site can be designed. The midline is also marked on the chin, neck and sternal furcula. The incision should be horizontal with about $3-4 \mathrm{~cm}$, being more extended to the side where the surgery will be performed. After dissection by planes, thyroid cartilage should be widely exposed. ${ }^{4}$

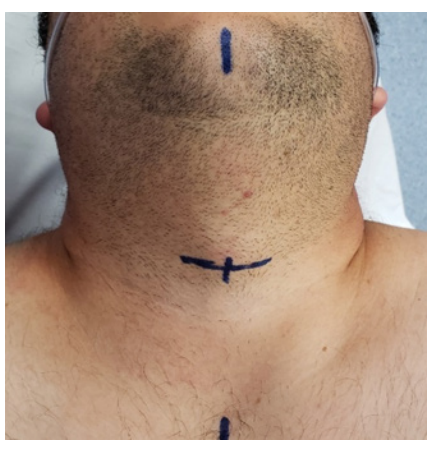

Source: Personal archive

The upper thyroid notch (A) and the midpoint on the lower margin of the thyroid cartilage (B) are then identified, the midpoint between the two described landmarks is measured $(\mathrm{C})$. The window is drawn from $4-5 \mathrm{~mm}$ from the midline and should be $4-5 \times 8-10 \mathrm{~mm}$ in women and $5-6 \times 10-12 \mathrm{~mm}$ in men. The perichondrium is incised, detached and removed. The window cartilage is removed with a scalpel and detacher, but if the patient is older and has more ossified cartilage, it is necessary to use a drill to make the window. The inner perichondrium of the thyroid lamina should be incised to gain access to the paraglottic space during medialization. An intact inner perichondrium limits the depth and precision of vocal fold displacement. . $^{2,4}$

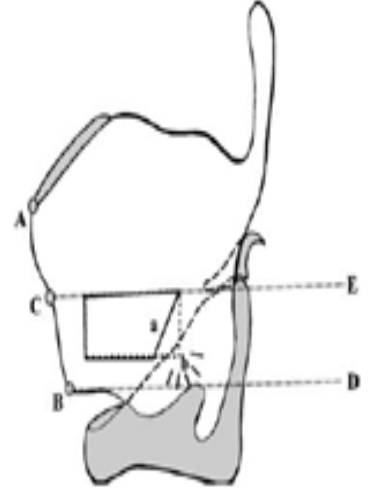


There are several materials that can be used to make the implant, even the cartilage window itself. Most surgeons use silicone (Silastic block or Montgomery ${ }^{\circledR}$ system) or Gore-Tex ${ }^{\circledR} .^{5,6,8}$ The thickness of the implant is approximately $4 \mathrm{~mm}$, refinements in its thickness are made from intraoperative voice monitoring.
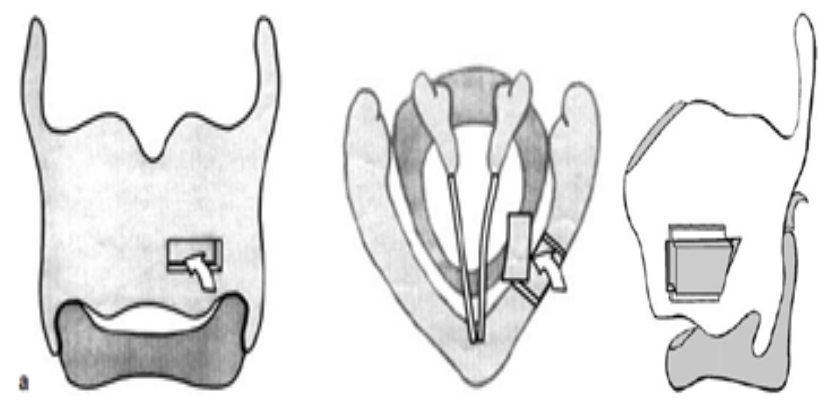

The implant can be fixed with nylon or prolene sutures or just be fitted. Closing by planes is then performed and the placement of a drain for 48 hours is optional. ${ }^{4}$
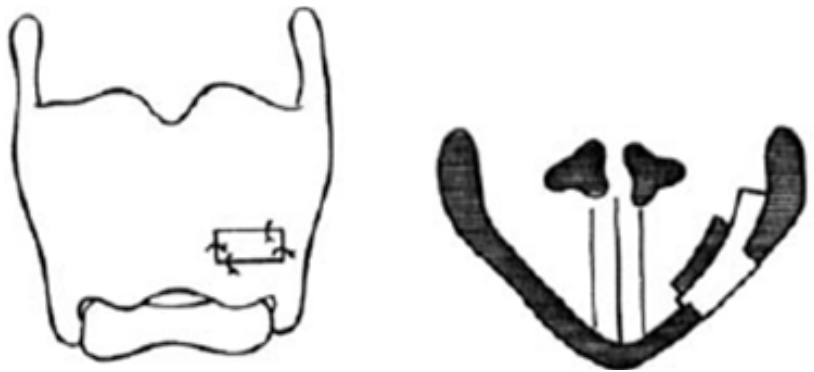

The main complications of this procedure are extrusion of the implant, edema of the laryngeal mucosa that can rarely lead to respiratory distress, bleeding, local infection and pharyngocutaneous fistula. ${ }^{2,6,7,9,10}$

\section{Adduction of arytenoids}

When the vocal process is medialized only on one side, it is usually performed in association with medialization thyroplasty. ${ }^{2,3,9,11}$ The lateral blade of the thyroid cartilage is exposed to its posterior margin, which can be rotated in the anteromedial direction for better visualization of the arytenoid region and its articulation with the cricoid. ${ }^{2,9}$

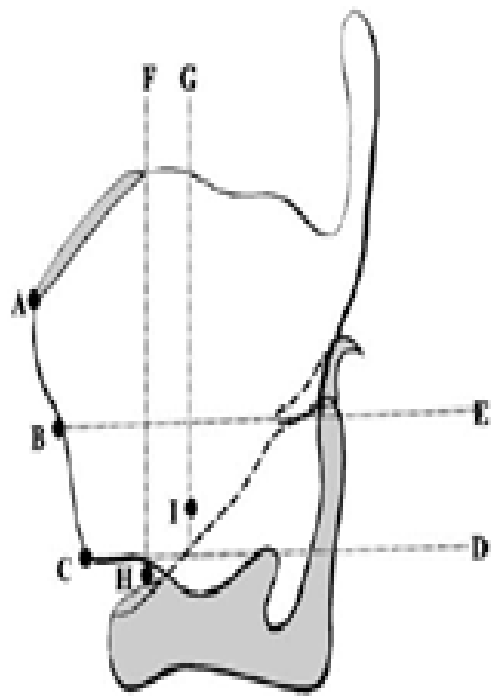

Usually, two sutures are performed. They cross the arytenoid muscle process and exit through the cricoarytenoid joint. As the recurrent laryngeal nerve is located posteriorly in the cricothyroid joint, it should be preserved whenever possible., ${ }^{2,9}$
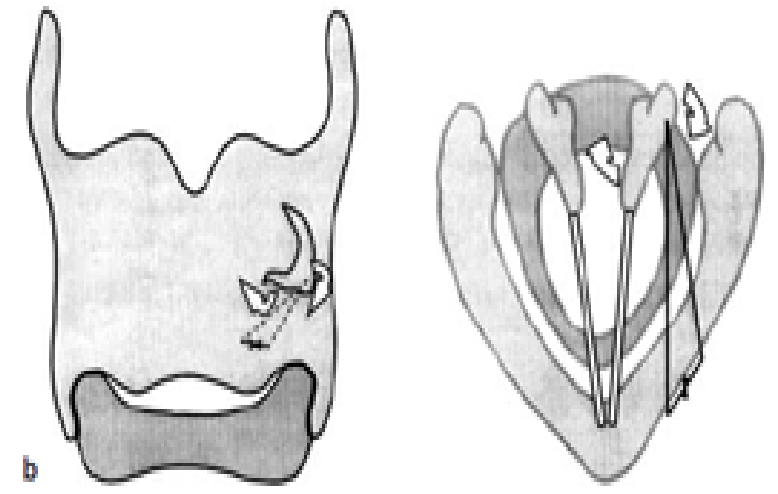

The main complication described is edema of the laryngeal mucosa. In cases where this surgery is performed, it is important to inform the patient that if future intubations are necessary, the anesthesiologist must be advised to use a smaller orotracheal tube. ${ }^{2}$

A new technique for endoscopic arytenoid adduction is being described by inserting two needles through the cricothyroid membrane, each with suture threads to perform the adduction of the muscular process. The disadvantage of performing this procedure in an endoscopic way is that it must be done under general anesthesia, so intraoperative voice monitoring is not possible. ${ }^{9}$

\section{Expansion laryngoplasty}

This procedure is indicated to increase the width of the glottis in cases where the vocal folds are hyper-induced, as in adduction spasmodic dysphonia. The purpose of this procedure is to improve voice quality, but not necessarily to improve breathing. ${ }^{1,2,12,13}$

\section{Lateralization thyroplasty (type II)}

The purpose of this procedure is to increase the transverse diameter of the thyroid cartilage, extending the glottic space. This procedure can be done through a lateral or medial approach, the latter being the most commonly used. ${ }^{1}$

\section{Lateral approach}

The exposure of the thyroid cartilage is done similarly as in medializationthyroplasty. A vertical incision is made in the lateral lamina of the thyroid cartilage and the fragment is mobilized, increasing the glottic space.

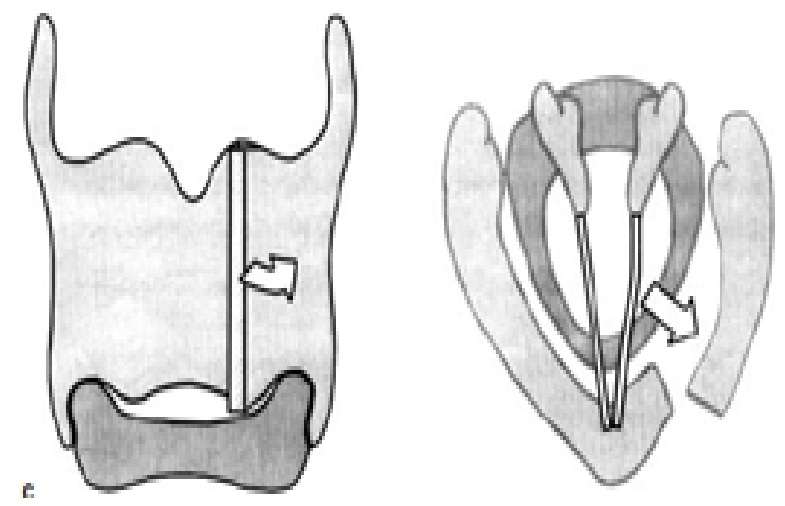




\section{Medial approach}

A vertical incision is made at the junction between the lateral layers of the thyroid cartilage. It is important to pay attention to the thyroepiglotic ligament, which should also be incised in the midline. ${ }^{2,13}$ Two silicone fragments with a width of 3-4 $\mathrm{mm}$ or two titanium bridges are placed superiorly and inferiorly to maintain the lateralization acquired with the incision. ${ }^{2,12,13}$ In the case of using titanium bridges, it is recommended to place a sternohyoid muscle flap to cover the perforation and decrease the dead space. ${ }^{12}$

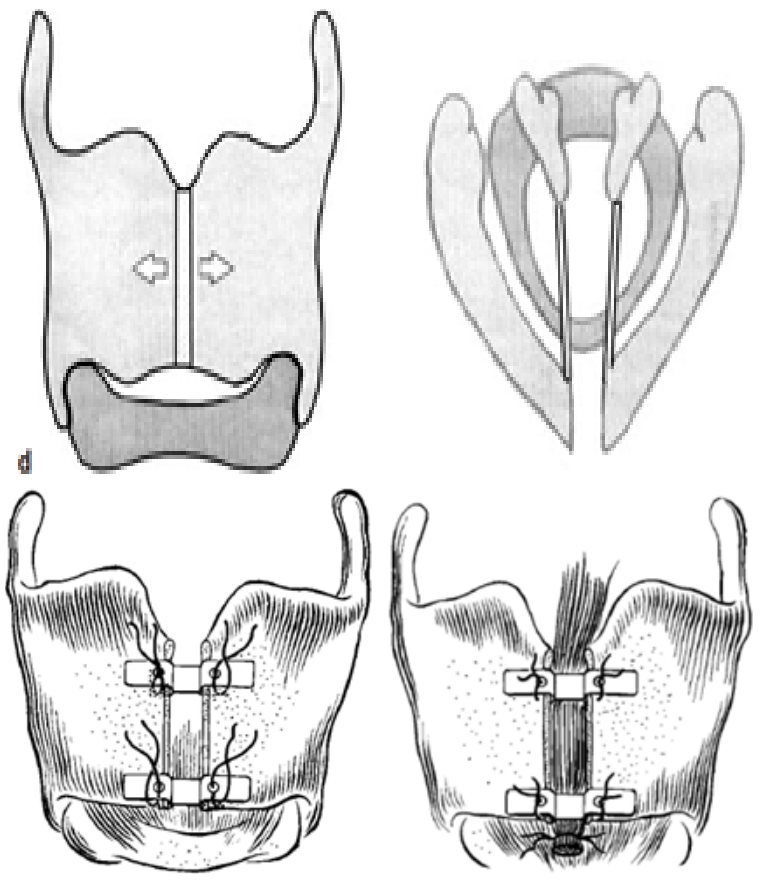

Complications associated with this procedure are local infection, perforation of the mucosa during the cartilage incision and, consequently, the formation of granuloma. ${ }^{13}$

\section{Vocal fold abduction}

It is performed directly through an intralaryngeal approach either by suturing the membranous and/or cartilaginous portion of the vocal fold laterally or by drying the vocal fold muscle.

\section{Relaxation laryngoplasty}

These procedures are indicated for stiff vocal folds such as in adduction spasmodic dysphonia or vocal sulcus, and in cases of inadequately acute voice due to mutational vocal disorders, in addition to cases of female-male transsexuality. ${ }^{1,14}$ The basic principle is to decrease the distance between the vocal fold insertions, thus the tension of the vocal folds.

\section{Shortening thyroplasty (type III)}

The exposure of the thyroid cartilage is performed in the same way as other aforementioned thyroplasty. A vertical cartilage tape (about $3-4 \mathrm{~mm}$ ) is outlined at the junction of the anterior third with the middle third of the lateral blade of the thyroid cartilage (about $7 \mathrm{~mm}$ distance from the midline). This procedure can be performed unilaterally or bilaterally. ${ }^{2,14}$ After removing the cartilage tape, two sutures of the remaining portions of the thyroid cartilage are performed for better correction. ${ }^{14}$
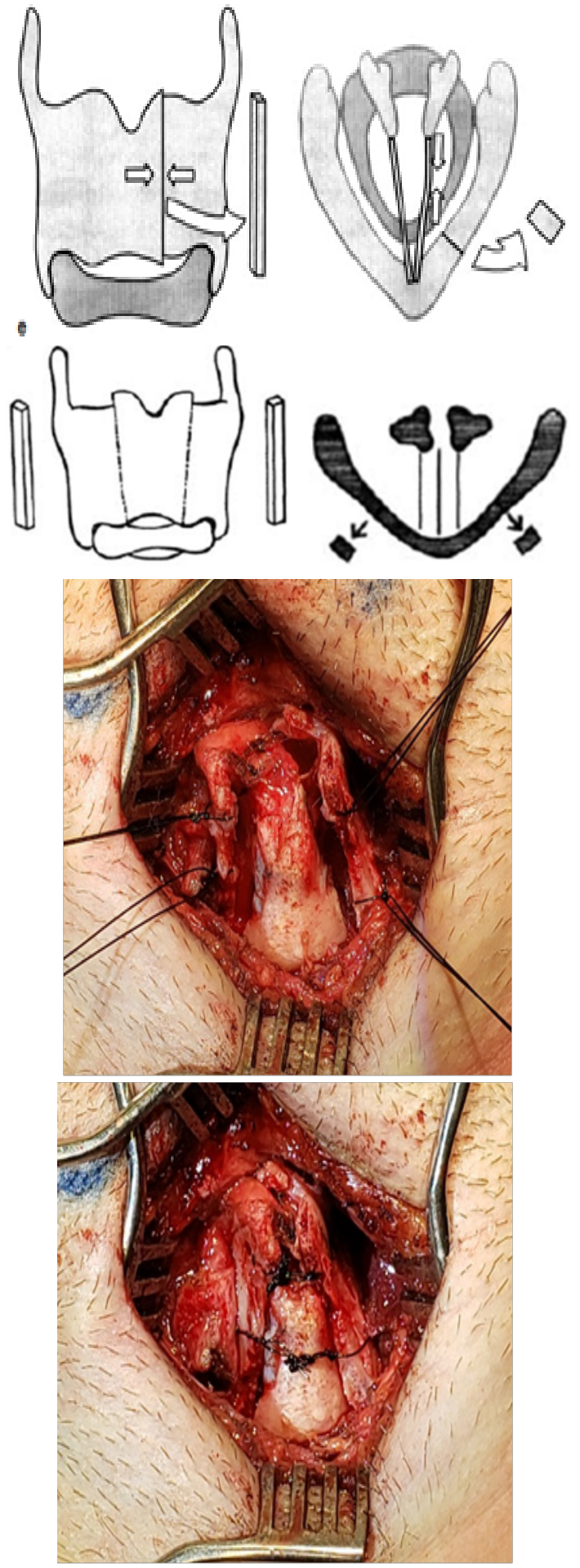

Source: Personal archive

\section{Tensioning laryngoplasty}

This technique is indicated in cases of curved vocal folds, such as in presbyphonia, bilateral lesion of the recurrent laryngeal nerve, and in cases of an inappropriately deep voice, such as in cricothyroid muscle paralysis, in androphony in women and in man-woman transsexuality. ${ }^{1,15}$ 


\section{Cricothyroid approach (type IV thyroplasty)}

The cricothyroid approach simulates the action of the cricothyroid muscle and substantially increases the vocal pitch. ${ }^{1}$ Once again, the exposure of the thyroid cartilage and the cricoid cartilage is performed similarly to the other described thyroplasty. Four sutures are performed so that the thyroid cartilage approaches the cricoid. ${ }^{2}$
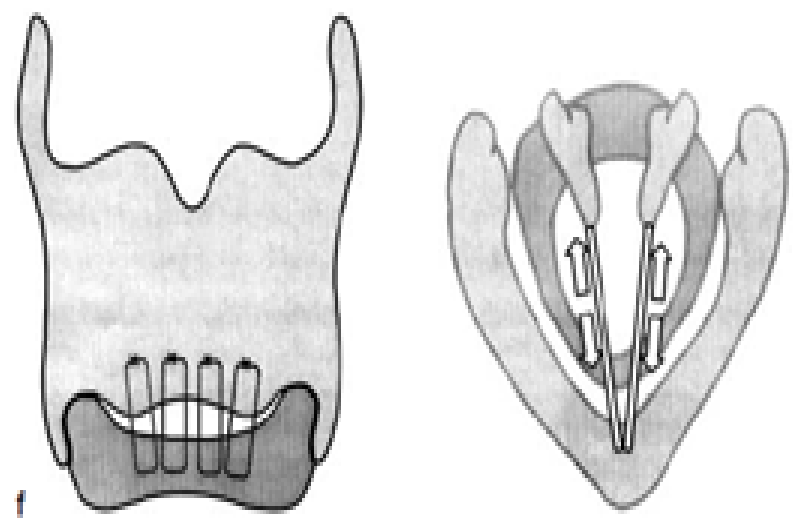

\section{Elongation thyroplasty}

In this type of surgery, in cases of medial approach, after exposure of the thyroid cartilage, implants are used and flaps are made in the middle portion of the thyroid cartilage. Both techniques aim to increase tension in the vocal folds.

Lateral approach
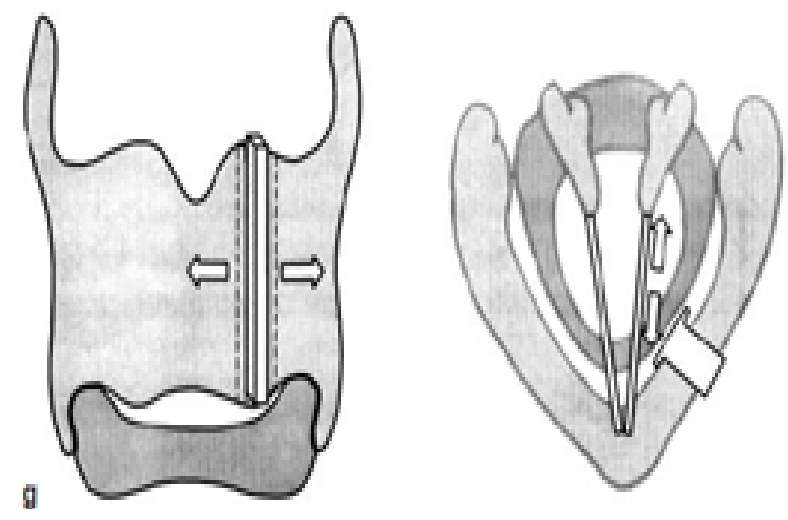

Medial approach
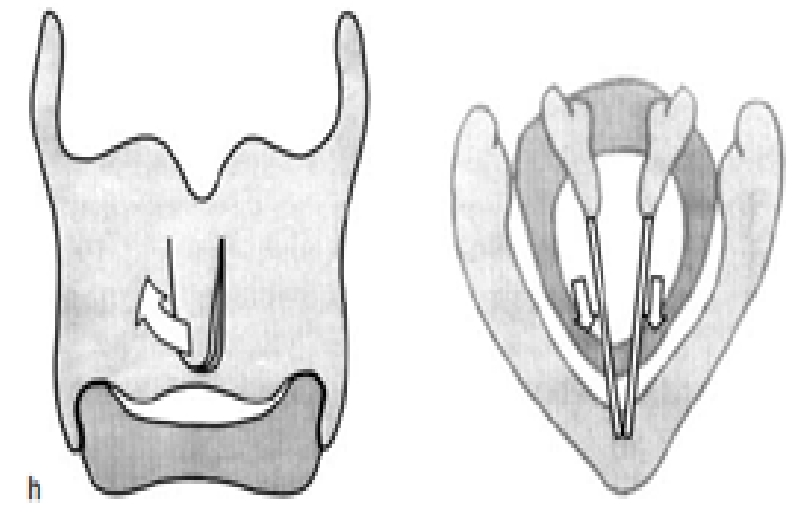

\section{Postoperative care}

As postoperative care, vocal rest for 5-10 days, use of antiinflammatories and antibiotics and avoid excessive neck movement for 10 days are recommended. Following surgery, post-operative voice therapy can improve patients' vocal discomfort, emotional responses and self-perception.In 2020, Laryngeal framework surgery approaches are remaining an important strategy in phonosurgery.

\section{Acknowledgments}

None.

\section{Conflicts of interest}

The author declares that there is no conflict of interest to disclose.

\section{Funding}

None.

\section{References}

1. G Friedrich, F I de Jong, H F Mahieu. et al. Laryngeal framework surgery: a proposal for classification and nomenclature by the Phonosurgery Committee of the European Laryngological Society. Eur Arch Otorhinolaryngol. 2001;258(8):389-396.

2. Hans F Mahieu. Practical applications of laryngeal framework surgery. Otolaryngol Clin N Am. 2006;39(1):55-75.

3. N Isshiki. Vocal mechanics as the basis for phonosurgery. The Laryngoscope, 1998;108(12):1761-1766.

4. T Ramadass, Nithya Narayanan, Girish N Kulkarni, et al. Thyroplasty type I - Apollo Hospital Experience, with a brief review of literature. Indian J Otolaryngol Head Neck Surg. 2003;55(3):180-183.

5. VyVy N Young, Thomas G Zullo, Clark A Rosen. Analysis of laryngeal framework surgery: 10-year follow-up to a national survey. The Laryngoscope. 2010;120(8):1602-1608.

6. Junlapan A, Sung K, Damrose EJ. Type I thyroplasty: a safe outpatient procedure. The Laryngoscope. 2019;129(7):1640-1646.

7. Nupur Kapoor Nerurkar, Shweta Mahadev Pawar, Shalaka Nilesh Dighe. A comprehensive 6-year retrospective study on medialization thyroplasty in the Indian population. Eur Arch Otorhinolaryngol. 2016;273(7):18351840 .

8. Anya J Li, Michael M Johns, Cristina Jackson-Menaldi, et al. Glottic closure patterns: type I thyroplasty versus type I thyroplasty with arytenoid adduction. $J$ Voice. 2011;25(3):259-264.

9. James J Daniero, C Gaelyn Garrett, David O Francis. Framework surgery for treatment of unilateral vocal fold paralysis. Curr Otorhinolaryngol Rep. 2014;2(2):119-130.

10. Tam S, Sun H, Sarma S, et al. Medialization thyroplasty versus injection laryngoplasty: a cost minimization analysis. J Otolaryngol Head Neck Surg. 2017;46(1):1-10.

11. Joseph Chang, Sarah L Schneider, James Curtis, et al. Outcomes of medialization laryngoplasty with and without arytenoid adduction. The Laryngoscope. 2017;127(11):2591-2595.

12. Nobuhiko Isshiki, Ichiro Yamamoto, Suminori Fukagai. Type 2 thyroplasty for spasmodic dysphonia: fixation using a titanium bridge. Acta Otolaryngol. 2004;124(3):309-312.

13. Matsushima K, Isshiki N, Tanabe $\mathrm{M}$, et al. Operative procedure of anterior commissure for type II thyroplasty. J Voice. 2018;32(3):374-380.

14. Yu Saito, Kazuhiro Nakamura, Shigeto Itan, et al. Type 3 thyroplasty for a patient with female-to-male gender identity disorder. Case Rep Otolaryngol. 2018;2018:1-4.

15. Gibbins N, Bray D, Harries Ml. Long-term quantitative results of an Isshiki type 4 thyroplasty - a case study. J Voice. 2011;25(3):283-287. 